\title{
The adipocyte component of bone marrow in heterotopic bone induced by demineralized incisor grafts
}

\author{
Krzysztof H. Włodarski ${ }^{1}$, Ryszard Galus ${ }^{1}$, Aniela Brodzikowska ${ }^{2}$, Paweł K. Włodarski ${ }^{1}$ \\ ${ }^{1}$ Department of Histology and Embryology, Center for Biostructure Research, \\ Medical University of Warsaw, Poland \\ ${ }^{2}$ Department of Conservative Dentistry, Institute of Stomatology, \\ Medical University of Warsaw, Poland
}

\begin{abstract}
The relative proportion of adipocytes to hematopoietic elements in the marrow of heterotopically induced bone evaluated 4-42 weeks post implantation of demineralized murine incisors was estimated by histological analysis of hematoxylin-eosin stained tissue sections. Using computerized image analysis of microphotographs, the proportion of nuclear cells vs. adipocytes was ascertained. The percentage of adipocytes in marrow increases over time. Such an effect, the replacement of myelopoietic marrow by adipogenic (yellow) marrow and the resorption of induced bone, is observed in human osteoporosis. A decline in the non-adipogenic cell compartments of bone marrow accompanying induced bone begins in the fourth week of induction, gradually progresses until the $26^{\text {th }}$ week, and does not change after that. The luminosity, a parameter used in image analysis and proportional to the number of nuclear cells, was $124 \pm 3$ in hematopoietic femoral bone marrow, and that of bone marrow of the induced bone was of a similar value (117 \pm 8$)$ in the fourth week. An evident decline in luminosity of bone marrow filling the foci of heterotopic bone was observed in samples taken at nine weeks $(82 \pm 20)$. This process progressed until the $26^{\text {th }}$ week, reaching a luminosity of $70 \pm 21$. At the $42^{\text {nd }}$ week, the luminosity remained at the same level $(71 \pm 27)$. This indicates that the replacement of hematopoietic bone marrow of heterotopically induced bone by unilocular adipocytes begins relatively early (the fourth week) and is persistent. (Folia Histochemica et Cytobiologica 2012, Vol. 50, No. 3, 444-449)
\end{abstract}

Key words: adipocyte, bone induction, bone marrow

\section{Introduction}

Foci of ectopic osteogenesis are colonized by myeloid tissue [1-3]. This tissue appears as early as two weeks post initiation of bone induction [3]. The cellular composition and function of heterotopic bone marrow have been analyzed by many authors. The

Correspondence address: P. K. Włodarski,

Department of Histology and Embryology,

Center for Biostructure Research,

Medical University of Warsaw,

Chalubinskiego Str. 5, 02-004 Warsaw, Poland;

tel.: + 48226281041 ext. 1418, fax: + 48226295282 ;

e-mail: pawel.wlodarski@wum.edu.pl; early work of Czerski and Zaleski, analyzing myelograms of ectopic bone plaques formed following intramuscular transplantation of urinary bladder mucosa in guinea pigs, showed a lack of significant differences between the 'induced' bone marrow, the host's femoral bone marrow, and the normal bone marrow of control animals [4].

Morphological analysis of the bone marrow occupying the foci of ectopic bone induced in mice by transplanted neoplastic cell lines has confirmed the presence of all types of myeloid cells, including megakaryocytes [5-8]. The infusion of 'induced' bone marrow into lethally irradiated recipient mice restored their hematopoiesis and saved the animals. The 'induced' bone marrow upon transplantation to irradi- 
ated animals formed spleen colonies of lympho-, granulo- and erytropoietic cells, and when cultured in vitro its stromal elements formed fibroblastic colonies. These fibroblast-like cells sometimes differentiated into adipocytes [9].

Stromal cells of bone marrow provide a niche for growth and differentiation of hematopoietic cells. Hematopoietic cells inhabit their own compartment. The stromal compartment of bone marrow contains fibroblasts, chondroblasts, osteoblasts and adipocytes. Progenitor cells for all types of stromal elements are termed 'mesenchymal stem cells'.

Adipocytes arise from mesenchymal stem cells [10], but recent data has revealed an additional source for preadipocytes - the hematopoietic stem cell precursors of the monocyte/macrophage lineage $[11,12]$.

Hematopoietic cells participate in the process of heterotopic osteogenesis, but their precise role is yet to be fully established [13]. On their own, they are unable to trigger the process of osteogenesis, but are present during its initial, preosteogenic, inflammatory stage, and are also present at the later stage of repopulation of osteogenic foci by myeloid tissue. Hematopoietic cells are absent during fibroblastic, chondrogenic and osteogenic stages of heterotopic bone induction [13].

The infiltration of adipocytes in osteoporotic patients' bone marrow suggests a regulatory function of bone marrow fat on the development of aged bone. Mesenchymal stem cells-derived adipocytes may inhibit osteoblast differentiation through TGF- $\beta$ mediated canonical Wnt signaling [14].

Adipogenesis in orthotopic normal bone marrow also increases with age in osteoporosis. There is an inverse correlation between bone loss and increment of marrow adipocyte content $[15,16]$. Also it has been shown that suppression of adipogenesis within bone marrow promotes bone formation [17].

In our previous work, during inspection of tissue sections of heterotopically induced bones at various stages of development, we noticed that the 'induced' bone marrow is overloaded with unilocular (yellow) adipocytes.

The current work was aimed at verifying this observation on a larger number of specimens. Thus, we investigated whether the proportion of adipocytic components of ectopic marrow is related to the duration of ectopic osteogenesis.

The rationale for such an investigation is a report on the expression of adipogenic markers by osteoblasts upon coculture of osteoblasts with adipocytes [18]. It suggests that bone marrow adipocytes are able to induce osteoblasts to differentiate into fat cells or that transdifferentiation of committed osteoblasts into adipocytes contribute to an increase in marrow fat content.

In addition we attempted to evaluate the dynamics of transition of hematopoietic marrow into an adipocytic one. Therefore, we attempted to determine the proportion of fat cells to other cellular elements of induced and of orthotopic bone marrow.

\section{Material and methods}

Balb/c mice, aged three months, bred and housed in our animal facility, were used in accordance with the Medical University of Warsaw guidelines for the care and use of laboratory animals. Heterotopic bone formation was induced in 51 healthy female mice by intramuscular implantation of demineralized for $20 \mathrm{hrs}$ in $0.6 \mathrm{~N} \mathrm{HCl}$ at $+4^{\circ} \mathrm{C}$ murine incisors according to the procedure described previously [19]. Under anesthesia, the implants were aseptically inserted into an intramuscular pocket executed in the thigh muscle by sharp scissors, following shaving of the surgery area. The wound was sutured by 3-0 Dexon "S" absorbable surgical suture and disinfected with $70 \%$ ethanol. At intervals following tooth matrix implantation, mice were sacrificed, the implants with surrounding tissues were excised, fixed in Bouin's solution overnight, demineralized in saturated disodium versenate dehydrate (EDTA) solution for 4 days and paraffin embedded. $7 \mu \mathrm{m}$ tissue sections were stained with hematoxylin-eosin.

Areas of bone marrow inside heterotopically induced bone were analyzed to determine the proportion of adipocytes relative to other elements of the marrow.

Intracortical bone marrow of the femur or tibia of the same animal was subjected to similar analysis as a control (orthotopic bone marrow).

The proportion of adipocytes in bone marrow in both orthotopic and heterotopic bones was quantified by analysis of histograms of microscopic images. Microphotographs were taken at a magnification of $400 \times$ with a digital camera using an Olympus C41 microscope and image acquisition software QuickPhoto Micro 2.2. (Promicra Ltd). Next, using Canvas X software (Daneba Inc.), images were analyzed as follows: area representing bone marrow was carefully selected with cursor omitting artefacts, converted to the negative, and the luminosity of this area only was analyzed. The hematoxylin-stained nuclei were bright, whereas unstained parts of the marrow section, such as the lumen of lipid drops in adipocytes or the lumen of sinusoids, were dark. The assumption was that the more adipocytes in the section, the lower the luminosity of the image. For the sake of simplicity, and because yellow adipocyte nuclei (if present in the section) are very small in proportion to the size of their lipid vacuole, the luminescence from all nuclei was ascribed to the hematopoietic compartment. Thus, luminescence of the analyzed image was in proportion to the ratio of hematopoietic cells vs. adipocytes. 
Table 1. Orthotopic and ectopic bone marrow luminosity as a function of age of ectopic bone foci

\begin{tabular}{|l|c|c|}
\hline $\begin{array}{l}\text { Duration of bone } \\
\text { induction } \\
\text { (weeks) }\end{array}$ & $\begin{array}{c}\text { Number of } \\
\text { specimens examined } \\
\text { (number of } \\
\text { sections analyzed) }\end{array}$ & $\begin{array}{c}\text { Mean } \\
\text { luminosity } \pm \text { SD }\end{array}$ \\
\hline 4 & $12(18)$ & $117 \pm 18$ \\
\hline 9 & $3(12)$ & $82 \pm 20$ \\
\hline 13 & $11(22)$ & $83 \pm 29$ \\
\hline 26 & $8(9)$ & $70 \pm 21$ \\
\hline 42 & $9(11)$ & $71 \pm 27$ \\
\hline $\begin{array}{l}\text { Femoral and tibial } \\
\text { bone marrow (control) }\end{array}$ & $8(15)$ & $124 \pm 3$ \\
\hline
\end{tabular}

To achieve consistent results, identical illumination and exposure conditions were used during the taking of photographs of each specimen. Erythrocytes filling the sinuses lumen gave also light illumination, and this illumination accounted for the luminosity of myeloid compartment.

Histograms of luminosity of the delineated areas of bone marrow were expressed as the mean value \pm SD.

The histograms of induced bone marrow were analyzed four, nine, 13, 26 and 42 weeks following incisor matrix implantation. The number of animals bearing induced bone (equal to the number of specimens examined) and the number of evaluations of histological sections of induced bone for each post-induction period assessed were pooled, and the mean values of luminosity for each time-group are presented in Table 1.

Statistical analysis. The significance of differences between luminosities was analyzed using ANOVA test with Scheffe's post hoc test. Statistical test was considered significant when the $\mathrm{p}$ value was less then 0.05 . Analysis was performed using SAS v.6.12 for Windows software (SAS Institute, USA).

\section{Results}

Implantation of demineralized syngeneic incisors into muscular pockets resulted in induction of cartilage and bone, both inside the chamber and on the surface of the implanted dentine matrix. Bone formation was preceded by cartilage formation, or bone was laid down immediately on the implanted tooth matrix. Thus the ectopic bone was formed by both endochondral and intramembranous ossification. Spaces delineated by newly-formed bone were colonized by bone marrow.

In our experimental system, applied bone marrow was observed as early as two weeks post bone induction [3]. On inspection of histological sections the 'induced' bone marrow was composed of hematopoietic cells, including megakaryocytes, and yellow adipocytes were present in varying numbers. No multilocular (brown) adipocytes were present in either ectopic or orthotopic (femoral) bone marrow.

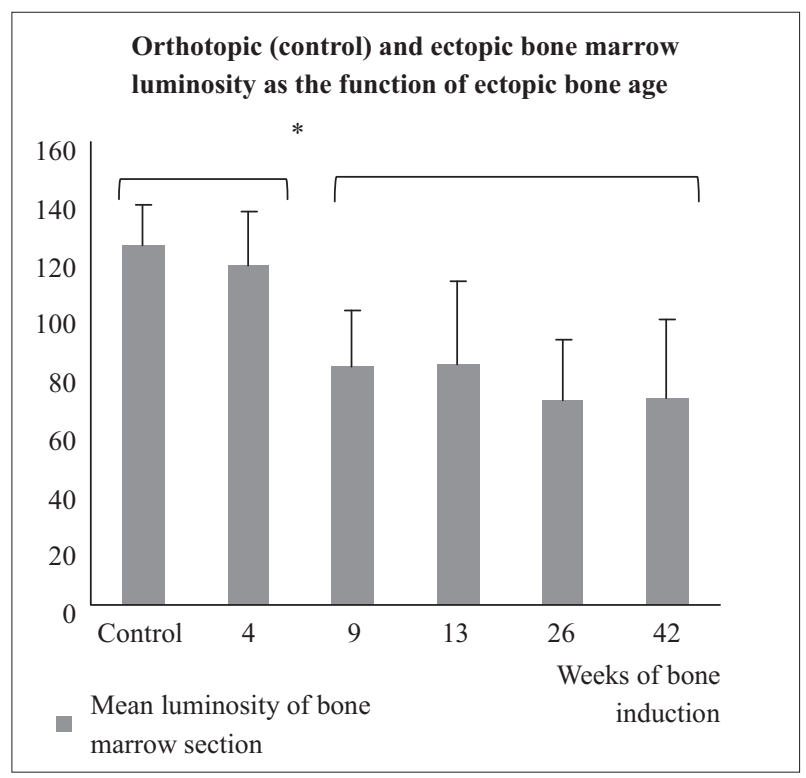

Figure 1. Orthotopic (control) and ectopic bone marrow mean luminosity as the function of ectopic bone age. Error bars represent $\mathrm{SD} ;$ * $\mathrm{p}<0.05$ between control and young (4 weeks) bone marrow of induced bone and older (9-42 weeks) induction by Student's-test

With an increase in age of induced bone, an increase in marrow adiposity at the expense of the hematopoietic component (Figure 2), and a decrease of luminosity, were observed (Figure 1, Table 1).

Evaluation of adiposity in the 'induced' bone marrow was performed at various stages of bone induction, 4-42 weeks post initiation of bone induction.

The yield of heterotopically induced bone, as evaluated by inspection of numerous sections of given samples, varied within a group of the same post-induction interval. However, the quantity of induced bone declined with the duration of bone induction. In the samples harvested 42 weeks post matrix implantation, only in nine out of 11 cases was a small quantity of induced bone found. This would suggest the complete resorption of heterotopically induced bone within one year.

No such changes occurred in the orthotopic bone marrow of tibiae or femora of the same animals.

A potential limitation of our approach is that all empty spaces, such as lumen of empty vessels and artifact-resulting cracks can influence luminosity. Also, the luminosity of erythrocytes accounts for the luminosity of a nuclear compartment. The greater the number of sections assessed, however, the less significant are these limitations.

\section{Discussion}

Heterotopic bone induced by implantation of demineralized tooth matrix is colonized by hematopoiet- 

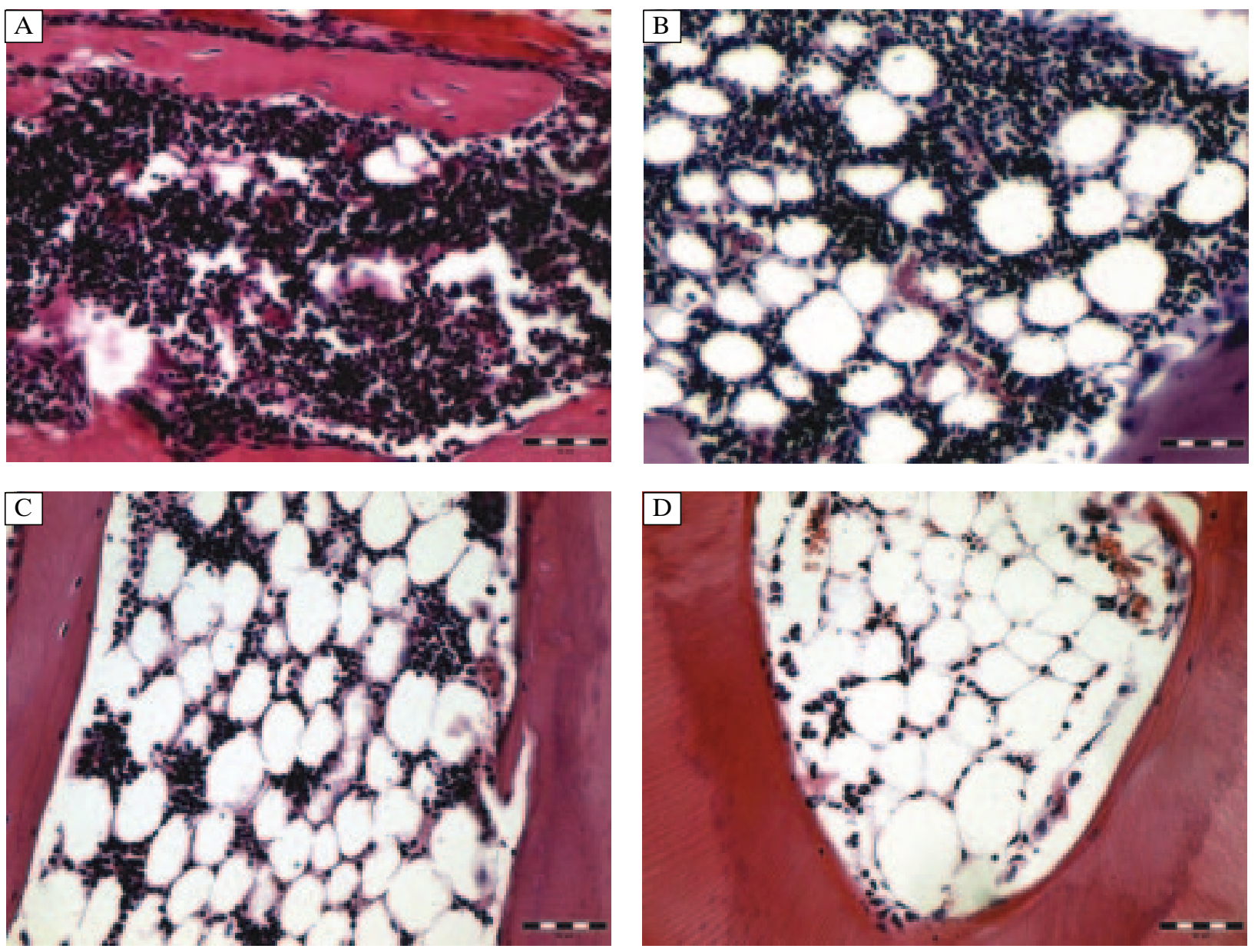

Figure 2. Histology and luminosity of bone marrow at $4^{\text {th }}(\mathbf{A}), 9^{\text {th }}(\mathbf{B}), 13^{\text {th }}(\mathbf{C})$, and $42^{\text {nd }}(\mathbf{D})$ week of ectopic bone induction. Scale bars are $50 \mu \mathrm{m}$. With aging of induced bone, the population of adipocytes in the marrow increases

ic bone marrow [3]. In the present work, we have analyzed whether the adipocyte content in such bone marrow changes over time from induction.

Adipocytes are the stromal components of bone marrow and in several instances their numbers increase in bone pathology [15-17]. Adipocytes and osteoblasts share a common precursor cell in the bone marrow stroma, termed the mesenchymal stem cell [16, 20-23]. A switch of differentiation of stromal stem cells from an osteogenic lineage into adipocytogenesis impairs bone formation by reducing the number of bone forming cells [22].

In osteoporosis, the number of adipocytes in bone marrow is significantly higher than in healthy subjects. It is postulated therefore that a contributory factor in osteoporosis is a diversion of activated mesenchymal stem cells from osteogenesis into adipocytogenesis $[16,17]$.

Bone marrow adipocytes are negative regulators of the hematopoietic microenvironment. Recent data points to the involvement of adipocytes in osteoclast differentiation and function [24]. Promotion of os- teoclastogenesis by adipocytes could explain an increased adipocyte component of bone marrow in osteoporosis, leading to bone resorption eventually.

Adipocyte-rich marrow is associated with a lower level of hematopoietic progenitors, since adipocytes hinder hematopoietic progenitor expansion following bone marrow transplantation into irradiated mice. Interestingly, this negative influence of adipocytes on a post-transplant hematopoietic infusion can be overcome if adipogenesis is blocked by inhibition of adipocyte transcription factor PPAR [25, 27].

Our present study provides information on the dynamics of transition of the red bone marrow into the yellow one in ectopic bones. This process is relatively fast and the change is persistent. The results presented here point to a substantial difference between the physiology of ectopic and orthotopic bone marrow. Heterotopically induced hematopoietic bone marrow lasts for a relatively short time, and within a few weeks converts into adipocytic marrow. This transition correlates with the degradation of ectopic bone. The induced ossicles are resorbed ex inactivatate [26]. 
We are unable to elucidate the reason for ectopic bone degradation; however, a lack of physical forces that stimulate ectopic bone has been blamed [26]. Skeletal muscles do not anchor to ectopic bones. There is an analogy between ectopic bone and tail vertebrae in mice. In contrast to the thoracic vertebrae, tail vertebrae are filled with adipocytic marrow [27], and only a small amount of muscles are attached to them, thus possibly they are not strongly bone stimulant. In contrast, bone exposed to muscle activity, such as tibiae, femora, or thoracic vertebrae, are filled with hematopoietic, red marrow.

Ectopic bones also do not function as an anchorage for skeletal muscles and, similarly to tail vertebrae, are also not stimulated to osteogenic activity by muscle-generated physical forces. In such circumstances there is no demand for ectopic bones to generate new osteoblasts or remodeling, and the stromal cells in their marrow switch their differentiation into adipocytes, instead of into osteoblasts. Adipocytes, in turn, promote osteoclastic differentiation, thus accelerating degradation of ectopic and aging bones [24, 28].

An increased marrow adipogenesis in the extraskeletal bones could either cause bone loss or be a result of bone immobility [29]. Experiments on coculture of osteoblasts with marrow adipocytes which have demonstrated transdifferentiation of osteoblasts into adipocytes by paracrine secretion speak in favor of the first possibility. Such transdifferentiation of committed osteoblasts into adipocytes may contribute to an increase in adipocytes at the expense of bone-forming cells [18]. On the other hand, prolonged immobility is associated with bone marrow adiposity when the inert bone loses its hematopoietic component, but the mechanism of activation of adipogenesis in the marrow of immobile bone is obscure [29].

Bones stimulated by mechanical forces are not degraded and their marrow is mostly hematopoietic. The findings presented here are in line with the clinical observation that immobilized bone becomes osteoporotic, and such a condition is combined with replacement of hematopoietic marrow into adipocytic marrow $[29,30]$.

The murine model of ectopic osteogenesis can be useful to study the interrelationship among hematopoietic, adipogenic and osteoblastic cells within the marrow. More precise determination of the developmental time course of adipocyte generation in the bone marrow of ectopic bones unexposed to mechanical forces will help in the understanding of the function of bone marrow adipogenesis and its role in the development of osteoporosis. This model can be applied for testing inhibitors of adipocyte differentiation in order to promote osteoblastic differentiation, a possible application in prevention and treatment of osteoporosis [17, 29, 30].

In conclusion, the presented data demonstrates the gradual conversion of hematopoietic bone marrow into adipocytic, yellow, marrow in ectopic, immobilized bone. They support a suggestion made by Gurewitch et al. that osteogenic and hematopoietic differentiation pathways compete for their common precursor [5], and such a switch is combined with immobilization and subsequent degradation of bone.

\section{Acknowledgements}

We thank Dr. Wynn Parry from the University of Liverpool for critiquing the manuscript, and Mrs. Ewa Wiśniewska for laboratory assistance.

\section{References}

1. Spellman RM, Marin H, Graham JH, Stefanini M. Heterotopic bone marrow production in the dog in response to transplantation of uroepithelium. J Urol. 1958;80:68-71.

2. Huggins $\mathrm{CB}$. The formation of bone under the influence of the urinary tract. Clin Orthop Rel Res. 1968;59:7-19.

3. Włodarski K. Heterotopic bone marrow formation in xenogeneic implants of insoluble bone matrix gelatin. Clin Orthop Rel Res. 1982;171:210-221.

4. Czerski P, Zaleski M. Bone marrow formation accompanying induced osteogenesis in guinea-pig. Haematologia Latina. 1962;5:33-43.

5. Gurewitch O, Slavin S, Feldman AG. Conversion of red bone marrow into yellow. Cause and mechanisms. Med Hypotheses. 2007;69:531-536.

6. Włodarski K, Jakóbisiak MI. Cellular composition of bone marrow derived from the heterotopic ossicles inducrd by xenogeneic epithelia of human amnion and dog transitional epithelium. Arch Immunol Therap Exp (Wroctaw). 1978;26:1027-1031.

7. Włodarski K, Jakóbisiak M. II. The concentration of hematopoietic stem cells in the induced bone marrow and its ability to repopulate lethally irradiated recipients. Arch Immunol Therap Exp (Wroctaw). 1978;26:1033-1036.

8. Włodarski K, Jakóbisiak M, Janowska-Wieczorek B. Heterotopically induced bone marrow formation: morphology and transplantation. Exp Hematol (Dallas). 1980;8:1016-1023.

9. Włodarski KH, Galus R, Włodarski PK. Osteogenic potential of bone marrow stromal cells. Ortop Traumatol Rehabil. 2006;8:573-577.

10. Laharrague P, Casteilla L. The emergence of adipocytes. Endocr Dev. 2010;18:21-30.

11. Sera Y, LaRue AC, Moussa O et al. Hematopoietic stem cell origin of adipocytes. Exp Hematol. 2009;37:1108-1120.

12. Majka SM, Fox KE, Psilas JC et al. De novo generation of white adipocytes from the myeloid lineage via mesenchymal intermediates is age, adipose depot, and gender specific. Proc Natl Acad Sci USA. 2010;107:14781-14786.

13. Kaplan FS, Glaser DL, Shore EM et al. Hematopoietic stem cell-contribution of ectopic skeletogenesis. J Bone Jt Surg Am. 2007;89:347-357.

14. Zhang $\mathrm{H}, \mathrm{Lu} \mathrm{W}, \mathrm{Zhao} \mathrm{Y}$ et al. Adipocytes derived from human bone marrow mesenchymal stem cells exert inhibitory effects on osteoblastogenesis. Curr Mol Med. 2011;116:489-502. 
15. Di Iorgi $\mathrm{N}$, Mo AO, Grimm $\mathrm{K}$ et al. Bone acquisition in healthy young females is reciprocally related to marrow adiposity. J Clin Endocrinol Metab. 2010;95:2977-2982.

16. Rodriguez JP, Astudillo P, Rios G et al. Adipogenesis and osteoporosis. Rev Med Chile. 2009;137:827-836.

17. Liu HY, Wu AT, Tsai CY et al. The balance between adipogenesis and osteogenesis in bone regeneration by plateletrich plasma for age-related osteoporosis. Biomaterials. 2011;32:6773-6780.

18. Clabaut A, Delplace S, Chauveu C et al. Human osteoblasts derived from mesenchymal stem cells express adipogenic markers upon coculture with bone marrow adipocytes. Differentiation. 2010;80:40-45.

19. Włodarski PK, Galus R, Włodarski KH, Brodzikowska A Heterotopic osteogenesis by murine demineralized incisors at lesions sites induced by Concanavalin A in mice. Connect Tissue Res. 2009;50:1-6.

20. Verma SR, Rajaratnam JH, Denton J, Hoyland AS, Byers RJ. Adipocytic proportion of bone marrow is inversely related to bone formation in osteporosis. J Clin Pathol. 2002;55:693-698 .

21. Menssen A, Haupi T, Sittinger M et al. Differential gene expresion profiling of human bone marow-derived mesenchymal stem cells during adipogenic development. BMC Genomics. 2011;12:461-502.

22. Weivoda MM, Hohl RJ. Geranylgeranyl pyrophosphate stimulates PPARgamma expression and adipogenesis through the inhibition of osteoblast differentiation. Bone. 2011 Oct 14 (Epub ahead of print).

23. Ichikawa J. Serum-free medium with osteogenic supplements induce adipogenesis in rat bone marrow stromal cells. Cell Biol Int. 2010;34:615-620.

24. Goto H, Hozumi A, Osaki M et al. Primary human bone marrow adipocytes support TNF-alpha induced osteoclast differentiation and function through RANKL expression. Cytokine. 2011;56:662-668.

25. Justenzen S, Stenderup K, Eriksen EF, Kassem M. Maintenance of osteoblastic and adipocytic differentiation potencial with age and osteoporosis in human marrow stromal cells culture. Calcif Tissue Int. 2002;71:36-44.

26. Kochanowska I, Włodarski K, Pieńkowski M, Ostrowski K. Influence of osteoprotegerin (OPG) on resorption of heterotopically induced ossicle. Cell Tissue Banking. 2004;5:125-128.

27. Naveiras O, Nard V, Wenzel PL et al. Bone marrow adipocytes as negative regulator of the haematopoietic microenvironment. Nature. 2009;460:259-263.

28. Duque G. Bone and fat connection in aging bone. Curr Opin Rheumatol. 2008;20:429-434.

29. Rosen CJ, Bouxsein ML. Mechanisms of disease: is osteoporosis the obesity of bone? Nat Clin Pract Rheumatol. 2006;2:35-43.

30. Rosen CJ, Ackert-Bicknell C, Rodriquez JP, Pino AM. Marrow fat and the microenvironment: developmental, functional, and pathological implications. Crit Rev Eukaryot Gene Expr. 2009;19:109-124.

Submitted: 13 September, 2011

Accepted after reviews: 20 February, 2012 